\title{
A national confidential enquiry into community acquired pneumonia deaths in young adults in England and Wales
}

J C G Simpson, J T Macfarlane, J Watson, M A Woodhead on behalf of the British
Thoracic Society Research Committee and Public Health Laboratory Service
Stepping Hill Hospital, Stockport SK7 2JE, UK J C G Simpson

Respiratory Medicine, City Hospital,

Nottingham NG5 1PB, UK

J T Macfarlane

PHLS Communicable Disease Surveillance Centre, 61 Colindale Avenue, London NW9 5EQ, UK

J Watson

\section{Department of}

Respiratory Medicine, Manchester Royal

Infirmary, Manchester

M13 9WL, UK

M A Woodhead

Correspondence to:

Dr M Woodhead

woodhead@

central.cmht.nwest.nhs.uk

Received 10 April 2000 Returned to authors 14 June 2000

Revised manuscript received

10 August 2000

Accepted for publication

22 August 2000

\begin{abstract}
Background-The aim of this study was to describe the frequency, causal pathogens, management, and outcome of a population of young adults who died from community acquired pneumonia (CAP). Methods-Pneumonia deaths in England and Wales in adults aged 15-44 were identified between September 1995 and August 1996. Patients with underlying chronic illness including HIV infection were excluded. Clinical details for each case were collected from the hospital and general practitioner records.

Results-Death from CAP was identified in 27 previously well young adults $(1.2$ per million population per year). Twenty were known to have consulted a GP for this illness. Nine received antibiotics before hospital admission. A causative pathogen was identified in 17 cases (Streptococcus pneumoniae in eight). Bacteraemia was present in seven. All patients who reached a hospital ward received antibiotics $(69 \%$ within two hours of admission). The British Thoracic Society antibiotic guidelines for severe CAP were followed in only 10 cases. Cardiac arrest at home or on arrival at hospital occurred in six cases, one of whom was successfully resuscitated. Of the remaining 21 patients, $71 \%$ had two or more markers of severe CAP. All 22 who were admitted reached an intensive care unit, but 11 of these required transfer to another hospital for some aspect of intensive care. One third of patients died within 24 hours of presenting to the hospital.
\end{abstract}

Conclusions-Death from CAP in previously fit young adults still occurs. While some deaths might be preventable by better patient management, most are unlikely to be preventable by current management practices.

(Thorax 2000;55:1040-1045)

Keywords: community acquired pneumonia; death; young adults

The aetiology and outcome of community acquired pneumonia (CAP) in adults in the UK has been documented in a number of studies in the community, ${ }^{1}$ hospital, ${ }^{2-4}$ and the intensive care unit..$^{5-7}$ Despite the availability of antibiotics active against the known causative pathogens, these studies have confirmed that death still occurs in a significant proportion of patients, ranging from less than $1 \%$ of those in the community ${ }^{1}$ to more than $50 \%$ of those who reach the intensive care unit. ${ }^{5-7}$ Although severe illness and death occur most frequently in the elderly and those with chronic underlying diseases, young or previously fit patients also die. In one study of 62 adults admitted with severe CAP to the intensive care unit, $32 \%$ were aged less than 44 years ${ }^{7}$; in another study of 453 adults with CAP admitted to hospital, five of the 26 who died were aged between 15 and 44 years. ${ }^{4}$ It is in this group of patients that death from pneumonia might be considered to be most likely to be preventable.

Patients may die as a result of overwhelming disease, or deficiencies in management, or a combination of these two factors. One small UK study suggested that management deficiencies were common in younger patients dying from $\mathrm{CAP}^{8}{ }^{8}$ and another from the USA suggested that preventable factors may be present in up to one quarter of deaths from pneumonia. ${ }^{9}$ Studies of severe pneumococcal infection have suggested that the fulminating nature of the disease is the main determinant of death. ${ }^{10}{ }^{11}$

This study describes a population of young adults, admitted to hospital for CAP, who died from the illness. Our goals were to identify how frequently this occurred, to describe the common causal pathogens, and to identify whether preventable factors were present during the course of the illness and its management.

\section{Methods}

Deaths from pneumonia in England and Wales were identified from death certificates by the Office of Population Censuses and Surveys (OPCS; subsequently Office for National Statistics (ONS)). At the time that the study protocol was developed, deaths were coded by the ONS from death certificate entries as "underlying cause of death" (UCD). This was determined by the application of "Rule 3" which meant that pneumonia would appear as UCD only if no chronic pre-existing disease also appeared on the death certificate. If a chronic disease such as ischaemic heart disease also appeared somewhere on the death certificate, then the chronic disease would be deemed to be the UCD and not pneumonia.

Before commencing the main study two pilot studies were performed. The first was to ensure that the UCD could pick out patients who died 
from CAP without the researcher being overwhelmed with entries where pneumonia only occurred as the natural end to some other chronic illness. The second was to develop a methodology whereby relevant data omitted from the death certificate-for example, that the patient had underlying AIDS-could be captured to ensure that the correct population of previously well younger adults was being studied.

\section{PILOT STUDY}

In 1986 alone, death entries were coded not only as UCD but also by all mentioned causes. This provided an opportunity to validate UCD. OPCS provided details of all deaths in that year in England and Wales in people aged 15-54 years which included ICD9 codes 480-487 (pneumonia). Age and sex and the ICD code of the initial (cause 1a on the death certificate) and the final UCD (after application of Rule 3) and the ICD code of each mentioned cause on the certificate were provided.

Of 3355 death certificates on which pneumonia appeared, pneumonia was mentioned but not considered to be the UCD in 2962 $(88 \%)$. "Neoplasms" (31\%) and "diseases of the nervous system and sense organs" (12\%) were the most common UCDs in this group. Of the $393(12 \%)$ cases where pneumonia was coded as the UCD, in $235(64 \%)$ this was the only entry on the death certificate. It was concluded that pneumonia as the UCD on the death certificate could be used to focus on the patients sought in this study.

PILOT STUDY 2

Copies of the death certificates of the 65 patients in the 15-44 age group who died between October and December 1990 in which the UCD was pneumonia were provided by the OPCS. For each a confidential questionnaire was sent to the clinician responsible during the final illness.

Replies were received for $53(82 \%)$. Twenty eight deaths were definitely and two were probably HIV related. The remaining 23 (43\%) appeared to be previously well young adults who had died solely as a result of pneumonia. Of the 30 HIV related deaths, $22(73 \%)$ spent their final illness in one of five centres recognised for the care of HIV related illnesses; none of the 23 suitable cases who had died only from pneumonia were managed in these centres.

It was concluded that, by both using the case questionnaire and excluding cases from the five centres mentioned above, the population of previously well young adults in whom death was caused by CAP could be identified.

\section{MAIN STUDY}

Death certificate entries for pneumonia in adults aged 15-44 in England and Wales in the 12 months from September 1995 to August 1996 were identified by ONS. Records were collected continuously and sent to one researcher (JCGS) each month so that suitable cases were known to the study group about 8-10 weeks after the death certificate had been completed. During 1995 the ONS stopped coding UCD. Since we had found UCD coding to be a robust and practical method for identification of appropriate cases, UCD using Rule 3 was applied by one researcher (MAW) where necessary with the assistance of the coding clerks at the ONS. Cases from the five centres identified in pilot study 2 as having a high incidence of deaths from AIDS related pneumonia were excluded.

Overall ethical approval for the study was first obtained from the Central Manchester local research ethics committee and then for each individual case from the appropriate local research ethics committee before data on that case were sought.

For each case a confidential questionnaire was sent to the hospital consultant responsible for the care of the patient in their final illness, followed by a telephone call when necessary. In the absence of exclusion factors-for example, previously undeclared HIV infection - the consultant was requested to forward the case notes or a copy of them to the researcher. Where case notes could not be released, the researcher visited the institution to inspect them. The GP records were requested from the appropriate FHSA.

\section{MICROBIOLOGY}

Bacteria were considered to be causative if identified by Gram stain and/or culture in samples taken within the first 24 hours of hospital admission from normally sterile sites or if found in pure culture in a purulent sample of sputum. Virus isolation, positive urine $L e-$ gionella antigen, a fourfold rise in paired antibody titres or a single titre of $\geqslant 256$ to viral or atypical respiratory pathogens were also accepted.

Case details were recorded on standard forms and the data were stored and analysed using dBase 3 .

\section{Results}

In the 12 months of the study pneumonia was recorded on the death certificate of 576 adults aged 15-44 in England and Wales; 454 (79\%) cases with other underlying diseases on the death certificate (Rule 3) were excluded, leaving 122 cases with pneumonia as the UCD for further study. A response to the questionnaire was received from 115 (95\%) of the consultants responsible for these cases in their final illness. The seven cases with no information were excluded and questionnaire information led to the exclusion of a further 88 cases because previously undisclosed underlying conditions (most commonly HIV infection) contributed to the final illness.

The study definition of "previously well young pneumonia death" was therefore fulfilled by 27 subjects ( $5 \%$ of the total). The population aged 15-44 in England and Wales at the time of the study was 22.5 million, giving an incidence of 1.2 young pneumonia deaths per million per year. Criteria for all 549 cases finally excluded are summarised in fig 1 . Underlying conditions leading to exclusion are shown in table 1. Only two included patients 


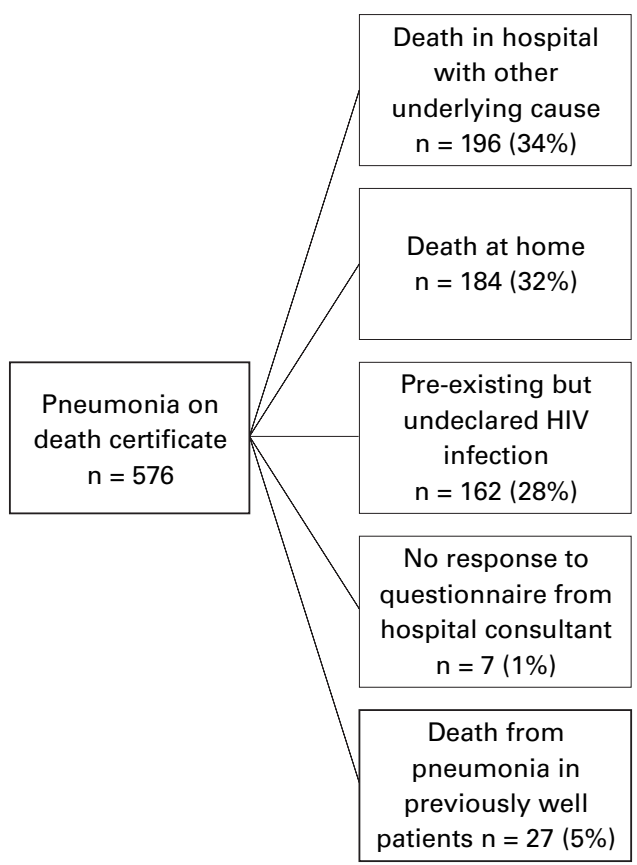

Figure 1 Categorisation of 576 patients with pneumonia recorded on death certificate.

Table 1 Underlying chronic diseases leading to exclusion of 196 cases admitted to hospital

\begin{tabular}{lr}
\hline Underlying disease & $\%$ \\
\hline Neurological & 35 \\
Chronic alcohol abuse & 18 \\
Neoplastic disease & 12 \\
Cardiac disease & 8 \\
Developmental abnormalities & 5 \\
Other & 22 \\
\hline
\end{tabular}

Table 2 Demographic data for the 27 previously well young subjects who died from pneumonia

\begin{tabular}{ll}
\hline Male & $11 / 27(41 \%)$ \\
Caucasian & $22 / 25(88 \%)$ \\
Mean (SD) age & $34(7.6)$ years (range \\
& $18-43)$ \\
$\begin{array}{c}\text { Median illness duration (before } \\
\text { hospital admission) }\end{array}$ & 5 days (range 2-14) \\
$\begin{array}{c}\text { GP consultation for this illness } \\
\text { before hospital admission }\end{array}$ & $20 / 27(74 \%)$ \\
\hline
\end{tabular}

were documented to have risk factors for HIV infection (homosexual contacts 5 and 10 years ago; both were HIV negative).

Relevant hospital records were available for all 27 cases and GP records for $16(59 \%)$ of them (no GP stated/notes lost $(n=5)$, no response to request $(n=5)$, GP records could not be accessed without permission from the next of kin $(n=1))$. Post mortem findings were known in 15 cases, not performed in two, and not known in 10 . The demographic data are shown in table 2 .

\section{MANAGEMENT BY GP}

Of the 20 cases who were known to have consulted a GP for this illness, $7 / 15(47 \%)$ had done so more than once and 15/20 (75\%) had seen the GP on the day of hospital admission. In nine the GP diagnosis did not suggest a respiratory infection (one diarrhoea and vomiting, one gastroenteritis, one musculoskeletal, one urinary tract infection, one cardiac arrest, four not known). A diagnostic label consistent with lower respiratory tract infection (LRTI) was applied in $11(55 \%)$ cases (five pneumonia, four LRTI, one chest infection, one "flu" with pleurisy), of whom five were recorded as being prescribed antibiotics. Four of the five antibiotic prescriptions (benzyl penicillin, aminopenicillin and/or macrolide) are considered to be an appropriate empirical choice for LRTI (the poor in vitro activity of ciprofloxacin against Streptococcus pneumoniae makes it an inappropriate first choice for LRTI). In two of those in whom LRTI was diagnosed, the GP consultation was on the day of admission but both self-referred to hospital later the same day, one suffering a cardiac arrest. Three of those with a GP diagnosis of LRTI had seen the GP on more than one occasion but had not been prescribed an antibiotic.

MICROBIAL AETIOLOGY

Seven different causative pathogens were identified in 17 cases $(63 \%$ of the total and $77 \%$ of the 22 who reached a hospital ward, table 3 ) with $S$ pneumoniae being the most frequent. Bacteraemia was present in seven $(26 \%$ of the total and $32 \%$ of those reaching a hospital ward). Dual infection was identified in only one patient (Staphylococcus aureus and $S$ pneumoniae). No pathogens were identified in the patients who had a fatal cardiac arrest on hospital arrival.

HOSPITAL MANAGEMENT

Intravenous antibiotics were given to 23 patients including all 22 who reached a hospital ward (excluding those who reached hospital but who died following cardiorespiratory arrest immediately on arrival). In nine of 13 (69\%) patients for whom documentation was available, this was given within two hours of hospital admission. The antibiotic recommendation for severe CAP given in the British Thoracic Society (BTS) guidelines ${ }^{12}$ was followed in only 10 cases, although a further three received these with additional antibiotics and two received antibiotic combinations which would be considered to have a comparable spectrum of antibacterial activity (table 4 ). Nine patients received corticosteroids for their illness.

All 22 were admitted to intensive care before death, 13 within 24 hours of admission and all within six days. No patient suffered an "unexpected" cardiac arrest on a general medical ward. All 11 interhospital transfers involved an aspect of intensive care. In the 10 cases for whom the reason was clearly documented, four were because of the lack of an ICU bed in the host hospital and six were for "specialist" care. The nature of the "specialist" care was not always clear, but during their stay 10 patients received renal dialysis and three extracorporeal membrane oxygenation (ECMO).

Cardiac arrest at home or on arrival at hospital occurred in six $(22 \%)$ cases, from which only one was successfully resuscitated. Of the remaining $21,15(71 \%)$ had two or more of three markers of illness severity identified in the BTS study of CAP in hospital ${ }^{4}$ (respiratory rate $>29 / \mathrm{min}$, diastolic blood pressure 
Table 3 Causative pathogens

\begin{tabular}{lcl}
\hline Pathogen & No of cases & Source of pathogen \\
\hline Streptococcus pneumoniae & 8 & $\begin{array}{l}\text { Blood (5), sputum (3), pericardial fluid (1), not } \\
\text { known (1) }\end{array}$ \\
Staphylococcus aureus & 3 & $\begin{array}{l}\text { Blood (1), pleural fluid (1), bronchoalveolar } \\
\text { lavage (1), sputum (1) }\end{array}$ \\
Legionella sp & 2 & Serology (2), urine antigen (2) \\
Influenza A & 2 & Bronchoalveolar lavage (1), serology (1) \\
Mycoplasma pneumoniae & 1 & Serology (1) \\
Escherichia coli & 1 & Blood (1) \\
Streptococcus faecalis & 1 & Pleural fluid (1) \\
No pathogen identified & 10 & \\
\hline
\end{tabular}

^In some the organism was identified from more than one source.

Table 4 Initial empirical hospital antibiotics

\begin{tabular}{ll}
\hline Antibiotic(s) & No of cases \\
\hline Cephalosporin + macrolide & 10 \\
Aminopenicillin + macrolide & 2 \\
$\beta$-lactam / $/$-lactamase inhibitor + macrolide & 2 \\
Cephalosporin & 2 \\
Benzyl penicillin + cephalosporin + macrolide & 1 \\
Cephalosporin + flucloxacillin & 1 \\
Flucloxacillin + cephalosporin + macrolide & 1 \\
$\beta$-lactam/ $\beta$-lactamase inhibitor & 1 \\
Cephalosporin + metronidazole & 1 \\
Benzyl penicillin + flucloxacillin + cephalosporin + macrolide & 1 \\
Azlocillin + gentamicin & 1
\end{tabular}

Table 5 Severity markers on admission for 21 cases (excluding those who had a cardiac arrest on admission)

\begin{tabular}{lrrl}
\hline Parameter & Yes & No & Not recorded \\
\hline Respiratory rate $>29$ breaths $/$ min & 10 & 6 & 5 \\
Diastolic blood pressure $<61 \mathrm{~mm}$ Hg & 10 & 11 & 0 \\
Blood urea $>7$ mmol/1 & 16 & 5 & 0 \\
$\mathrm{Multilobar}$ or bilateral radiographic consolidation & 5 & 15 & 1 \\
$\mathrm{WaO}_{2}<8 \mathrm{kPa}( \pm$ supplemental oxygen $)$ & 11 & 8 & 2 \\
Albumin $<30 \mathrm{~g} / 1$ & 12 & 9 & 0 \\
Arterial $\mathrm{pH}<7.35$ & 4 & 17 & 0 \\
& 6 & 11 & 4 \\
\hline
\end{tabular}

$<61 \mathrm{~mm} \mathrm{Hg}$, urea $>7 \mathrm{mmol} / \mathrm{l})$. Lack of documentation of the respiratory rate in five patients limited the value of retrospective severity assessment (table 5). There was no record of measurement of arterial blood gas tensions or the administration of oxygen in two patients.

OUTCOME

The median length of hospital stay was 8.5 days (range 1-36). Five patients died within 24 hours of admission to the ICU which means that, together with the five fatal cardiac arrests just before or after hospital admission, 10 of the 27 patients $(37 \%)$ died within 24 hours of hospital presentation.

\section{Discussion}

This study for the first time gives a measure of the true frequency of death from CAP both overall and in young, previously fit adults admitted to hospital. The 576 death certificate entries for pneumonia translates into an incidence of 25.6 per million per year. The frequency of 1.2 per million per year for previously fit young adults indicates that this is rare, but that such adults still die from CAP. The careful methodology applied in this study, together with the very high response rate to the questionnaire to admitting physicians, has allowed us both to identify and study previously fit patients with CAP who died in hospital and to separate them from those with underlying disease (including HIV infection).
The methods which we used for case identification confirmed the inaccuracy of death certificate entries. We sought to avoid the need to inspect the case notes of all 576 cases, which might have been more accurate but would have been time consuming and inefficient, while at the same time precisely identifying those with CAP without underlying disease who died in hospital. The careful evaluation of the use of $\mathrm{UCD} /$ Rule 3 in the two pilot studies supports the robustness of this approach and indicates that an analysis based solely on death certificates where pneumonia was mentioned would have produced a falsely high incidence. While it is possible that cases were missed by exclusion of all cases from the five hospitals recognised as centres for the care of HIV related illness and of the seven cases for whom there was no response from the hospital consultant, these numbers are likely to be so small as to have no material effect on the results.

It seems unlikely, but cannot be disproved, that unrecognised HIV infection could be a contributing factor in the 27 cases included in the final analysis as none of these cases was documented as having risk factors for such infection.

It was not part of the study design to investigate pneumonia deaths occurring outside hospital. Only nine unexpected deaths from CAP at home were recorded over one year in a population of 800000 in Nottingham and all cases were aged $>60$ years. ${ }^{13}$ It seems unlikely that a previously fit adult with severe pneumonia would not consult a GP and be referred to hospital, which suggests that the high number of deaths at home predominantly represents patients with underlying disease in whom death was expected.

The spectrum of underlying diseases identified in those excluded from the main study comprised predominantly neurological and neoplastic conditions, as expected. The presence of chronic alcohol related problems in $18 \%$ of cases was a second surprising finding in the study. A relationship between alcohol and pneumonia has long been recognised, but high alcohol intake has not previously been found to be common in UK studies of CAP. In these studies the frequency varies from "no cases"2 to $14 \% .^{3}$ No cases were identified in an earlier study of deaths from CAP in young adults in hospital. ${ }^{8}$ Lack of standardisation of a history of alcohol consumption or of a definition for high intake/alcoholism between studies makes data interpretation difficult. In two Spanish studies those with a high alcohol intake were found to be 3.9 (95\% CI 1.4 to 10.6$)^{14}$ and 5.23 (95\% CI 1.43 to 19.2$)^{15}$ times more likely to have CAP than a control group. The importance of the relationship between alcohol intake and pneumonia requires further study.

\section{MICROBIAL AETIOLOGY}

A causative pathogen was identified in a very high proportion of cases compared with the $26 \%$ of cases in whom an organism is found in routine management ${ }^{16}$ and the $36 \%$ found in another study of pneumonia deaths in young adults. ${ }^{8}$ This may be because a more detailed 
investigation was undertaken in these young sick patients or because a high proportion were bacteraemic, a marker of the fulminating nature of their illness. UK studies of adults admitted to hospital with CAP have found bacteraemia in $<10 \%$ of cases. ${ }^{2-4}$ This statistic must be interpreted with caution. One third of patients died within 24 hours of admission to hospital which suggests that, in many cases, microbiological information on which to base antibiotic prescription was not available before death. The spectrum of pathogens identified is similar to that in other UK studies of patients with severe CAP, with $S$ pneumoniae being the most frequent cause..$^{5-7}$

\section{MANAGEMENT BY GP}

Information regarding patient management by the GP is limited as the GP records were available for only just over half of the cases and useful information contained within them was scant. Nevertheless, three quarters of patients were known to have seen the GP for this illness, few had a correct diagnosis, and few had received antibiotics. This suggests that opportunities for early antibiotic administration may have been missed (albeit only by a few hours, but crucial hours in the context of what followed). Misinterpretation of the heterogeneous and non-specific presentations of pneumonia may have been the reason why antibiotics were not prescribed. It is possible that, in the two cases seen by a GP and who then self-referred to hospital, markers of illness severity were missed. This needs to be interpreted in the context of general practice where LRTIs are extremely common and are seldom severe. We would recommend that if a GP recognises severe pneumonia, even in a previously well young adult, urgent hospital referral should be made after administration of an antibiotic. Parenteral penicillin (or, if not available, oral amoxicillin $1 \mathrm{~g}$ stat) is recommended, as for suspected meningitis, and will provide initial cover for pneumococcal infection. There is a good argument to suggest that, when a GP decides to refer to hospital any patient with suspected CAP, they should start antibiotic treatment if the admission process is likely to delay the antibiotics by more than two hours. Studies have shown that it may take six hours or more for patients with non-severe CAP to receive antibiotics after admission to hospital, ${ }^{16}$ and other studies suggest that such delays may affect the outcome. ${ }^{17} 18$

There are no severity prognostic rules or guidelines that have been validated in the community. However, three simple clinical parameters (new mental confusion, raised respiratory rate $\geqslant 30 / \mathrm{min}$, low diastolic blood pressure $\leqslant 60 \mathrm{~mm} \mathrm{Hg}$ ) are well established as independent risk factors for death. The presence of two of these factors is associated with an increased chance of death of up to 20 times, and patients displaying these features should be regarded as having severe CAP and be admitted urgently. It seems sensible to suggest that the presence of even one adverse prognostic factor should trigger concern and the consideration of admission. ${ }^{419} 20$
RECOGNITION AND MANAGEMENT OF SEVERE ILLNESS

The frequency of presentation to the GP on the day of admission (75\%), cardiac arrest at home or on arrival at hospital (six cases), and admission to the ICU within 24 hours of hospital admission and death within 24 hours of hospital admission (10 cases) indicate that the illness was fulminating in up to half of the cases in this study. Whether cardiac arrest could have been prevented by immediate fluid or gas exchange management is not known. For those who did not die during the hospital admission process, general pre-ICU management was of a high standard. Severity marker recording was almost complete (with the exception of respiratory rate in five and blood gas tensions in two). Appropriate empirical parenteral antibiotics were given to three quarters of patients and were started within two hours of admission in two thirds. Easy access to CAP management guidelines for doctors working in medical assessment units, emphasising the recognition and immediate and appropriate management of severe CAP, would be beneficial and it is to be hoped that new UK national CAP management guidelines will help in this regard.

All were admitted electively to intensive care, and not only after an unexpected cardiac arrest on the general ward. The need to transfer to another hospital for specialist intensive care in 11 patients is of concern, and presumably reflects a local lack of expertise or intensive care facilities or beds, but it is not possible to determine from the data available whether this had an impact on the outcome. Attention has recently been drawn to underassessment of the severity of CAP if not performed in a structured way $^{19}$ and the poor quality of pre-ICU management in a high proportion of those with severe illness of diverse aetiology. ${ }^{21}$ For the 27 patients described here, it is unlikely that improvement in the pre-ICU management would have had any impact on the outcome.

\section{CONCLUSION}

Death from CAP in previously fit young adults is uncommon, but still occurs. The causative pathogens conform with the spectrum of organisms known to cause severe CAP and the illness should be appropriately treated empirically by the antibiotics recommended for CAP in the BTS guidelines. ${ }^{12}$ In a significant proportion the illness is very rapidly progressive and may not be curable even with optimum antibiotic therapy and appropriate intensive care support. While management improvements should always be sought, improvement in in-hospital management as currently practised is unlikely to change the course and outcome in anything other than a few cases. Antibiotics should be started as early as possible and it is recommended that they be given by the GP when referring such patients to hospital. It is only with advances in our understanding of what leads to such fulminating illness and treatments designed to reverse these processes that improvements in outcome are likely to follow. 
The study was funded by the Department of Health through the Royal College of Physicians Research Unit.

1 Woodhead MA, Macfarlane JT, McCracken JS, et al. Prospective study of the aetiology and outcome of Prospective study of the aetiology and outco
pneumonia in the community. Lancet 1987;i:671-4.

pneumonia in the community. Lancet $1987 ; 1: 671-4$.
2 White RJ, Blainey AD, Harrison KJ, et al. Causes of pneumonia presenting to a district general hospital. Thorax 1981;36:566-70

3 Macfarlane JT, Finch RG, Ward MJ, et al. Hospital study of adult community-acquired pneumonia. Lancet 1982;ii: 255-8

4 British Thoracic Society and Public Health Laboratory Service. Community-acquired pneumonia in adults in British hospitals in 1982-1983: a survey of aetiology, mortality, prognostic factors and outcome. $Q \mathcal{F}$ Med 1987;62: 195-220.

5 Woodhead MA, Macfarlane JT, Rodgers FG, et al. Aetiology and outcome of severe community-acquired pneumonia. $\mathcal{F}$ Infect 1985;10:204-10.

6 Hirani NA, Macfarlane JT. Impact of management guidelines on the outcome of severe community acquired pneumonia. Thorax 1997;52:17-21.

7 British Thoracic Society Research Committee and Public Health Laboratory Service. The aetiology, management Health Laboratory Service. The aetiology, management the intensive care unit. Respir Med 1992;86:7-13.

8 Tang CM, Macfarlane JT. Early management of younger adults dying of community acquired pneumonia. Respir Med 1993;87:289-94

9 Dubois RW, Brook RH. Preventable deaths: who, how often, and why? Ann Intern Med 1988;109:582-9.

10 Austrian R, Gold J. Pneumococcal bacteraemia with especial reference to bacteraemic pneumococcal pneumonia. Ann Intern Med 1964;60:759-66.
11 Hook EW, Horton CA, Schaberg DR. Failure of intensive care unit support to influence mortality from pneumococcal bacteremia. $\mathcal{F} A M A$ 1983;249:1055-7.

12 British Thoracic Society. Guidelines for the management of community-acquired pneumonia in adults admitted to hospital. Br f Hosp Med 1993;49:346-50.

13 Woodhead MA. Studies on pneumonia in the community and in hospital in Nottingham. Thesis/Dissertation, University of Nottingham, 1988.

14 Ruiz M, Ewig S, Torres A, et al. Severe community-acquired pneumonia. Risk factors and follow-up epidemiology. $A m \mathcal{F}$ Respir Crit Care Med 1999;160:923-9.

15 Fernandez SJ, Junque A, Estruch R, et al. High alcohol intake as a risk and prognostic factor for communityacquired pneumonia. Arch Intern Med 1995;155:1649-54.

16 Woodhead MA, Arrowsmith J, Chamberlain WR, et al. The value of routine microbial investigation in communityacquired pneumonia. Respir Med. 1991;85:313-7.

17 Meehan TP, Fine MJ, Krumholz HM, et al. Quality of care, process, and outcomes in elderly patients with pneumonia. fAMA 1997;278:2080-4.

18 Heath $\mathrm{CH}$, Grove DI, Looke DF. Delay in appropriate therapy of Legionella pneumonia associated with increased mortality. Eur f Clin Microbiol Infect Dis 1996;15:286-90.

19 Neill AM, Martin IR, Weir R, et al. Community acquired pneumonia: aetiology and usefulness of severity criteria on admission. Thorax 1996;51:1010-6.

20 Lim WS, Lewis S, Macfarlane JT. Severity prediction rules in community acquired pneumonia: a validation study. Thorax 2000;55:219-23.

21 McQuillan P, Pilkington S, Allan A, et al. Confidential inquiry into quality of care before admission to intensive are. BMF 1998;316:1853-8. 